\title{
A PROPERTY OF DERIVATIVES
}

\section{J. A. CLARKSON}

If a real function defined over a closed interval $[a, b]$ is differentiable at each point of the interval, it is well known that its derivative possesses the Darboux property: if $f^{\prime}(c)<\xi<f^{\prime}(d)$, then there is a point $e$ between $c$ and $d$ with $f^{\prime}(e)=\xi$.

Now let $\alpha, \beta$, with $\alpha<\beta$, be any two fixed reals, and consider the set $E(\alpha, \beta)=E\left\{x / \alpha<f^{\prime}(x)<\beta\right\}$. It is easily seen, as a consequence of the Darboux property, that any such set $E(\alpha, \beta)$ must contain a continuum of points, unless it is empty. The question of the measure of $E(\alpha, \beta)$ does not seem to be covered in the literature, except in the case in which the given interval is either $(-\infty, \beta)$ or $(\alpha,+\infty)$. We prove that any such set $E(\alpha, \beta)$ is either empty or of positive measure.

We remark that this result cannot be deduced from the Darboux property alone; Lebesgue exhibited a function ${ }^{1}$ which possesses that property without satisfying the measure condition. Another example is the following. Let $C$ be the Cantor closed nondense set of measure zero and power $c$ in the unit interval, and let $\left\{T_{n}\right\} \quad(n=1,2$, $3, \cdots)$ be a sequence of linear transformations such that the sets $T_{n}(C)$ are disjoint, and such that any sub-interval of $[0,1]$ contains some $T_{n}(C)$. We take $T_{1}$ to be the identity. Let the function $g(x)$ be defined on $C$ in such a way as to assume all values from zero to one inclusive; on $T_{n}(C)$ let $g(x)=g\left(T_{n}^{-1}(x)\right)$. On all remaining points of the unit interval set $g(x)=0$. It is clear that this function $g$ possesses the Darboux property, but that the set $E\{x / 1 / 2<g(x)<1\}$ will be nonvoid and of measure zero.

THEOREM. If $f(x)$ is real and everywhere differentiable in the closed interval $[a, b]$, then for any two reals $\alpha, \beta(\alpha<\beta)$, the set

$$
E(\alpha, \beta)=E\left\{x / \alpha<f^{\prime}(x)<\beta\right\}
$$

is empty or of positive measure.

Proof. We start with the following known result: ${ }^{2}$ if a continuous function $f(x)$ is differentiable in the interval $[a, b]$, with the possible exception of a denumerable set of points $x$, and if $f^{\prime}(x)$ is non-negative almost everywhere, then $f(x)$ is nondecreasing. It follows that if $f^{\prime}(x)$ exists for all $x$ in $[a, b]$, and $f^{\prime}(x) \geqq \lambda\left[\right.$ or $\left.f^{\prime}(x) \leqq \mu\right]$ for almost all $x$,

Received by the editors July 9, 1946.

${ }^{1}$ Lebesgue, Leçons sur l'intégration, 2d ed., Paris, 1928, p. 97.

2 Saks, Thêorie de l'intégrale, Warsaw, 1933, p. 141. 
then $f^{\prime}(x) \geqq \lambda\left[f^{\prime}(x) \leqq \mu\right]$ for all $x$ in $[a, b]$ (by considering the functions $f-\lambda x, f-\mu x)$. In other words, if a function is differentiable everywhere, then the least upper and greatest lower bounds of its derivative are not changed if sets of measure zero are neglected in computing them: this is the theorem which results from our formulation if either $\alpha=-\infty$ or $\beta=+\infty$. We shall make use of this remark in the following argument.

Consider $\alpha, \beta$ arbitrary and finite: write $E$ for $E(\alpha, \beta)$. Suppose $E$ not void, and $m E=0$; we shall show that a contradiction results. We write $E_{\alpha}=E\left\{x / f^{\prime}(x) \leqq \alpha\right\}, E_{\beta}=E\left\{x / f^{\prime}(x) \geqq \beta\right\}$, so that the interval $[a, b]=E+E_{\alpha}+E_{\beta}$, the three sets being disjoint.

First, $E \subset E_{\alpha}^{\prime} \cdot E_{\beta}^{\prime}$. To prove this relation, assume the contrary and let $x_{0}$ belong to $E$ but not to $E_{\alpha}^{\prime}$; then $x_{0}$ is in the interior of some interval $U, U \cdot E_{\alpha}$ void. But then in the interval $U$ the set of points where $f^{\prime}<\beta$ is not empty, since it contains $x_{0}$, but is of measure zero, since it is a subset of $E$. This contradicts the special case of our theorem which was referred to in our preliminary remark. Thus $E \subset E_{\alpha}^{\prime}$, and by a similar argument, $E \subset E_{\beta}^{\prime}$ also.

The derivative $f^{\prime}(x)$ belongs to Baire's first class, and hence, by a known theorem, ${ }^{3}$ if $A$ is any subset of $[a, b]$, and $f^{\prime}$ be considered for the moment on the domain $A$ alone, its points of discontinuity must form a set of the first category relative to $A$. Take for the subset $A$ the closure of our set $E$ : this set, $\bar{E}$, being closed, is of second category relative to itself, and hence we shall have a contradiction if we show that $f^{\prime}(x)$ is everywhere discontinuous considered on the domain $\bar{E}$. This may be seen as follows: let $x_{0}$ be a point, first, of $E$ itself. Since $E \subset E_{\alpha}^{\prime} \cdot E_{\beta}^{\prime}$, we have for any interval $I$ which contains $x_{0}$

$$
\sup _{x \in I} f^{\prime}(x) \geqq \beta, \quad \inf _{x \in I} f^{\prime}(x) \leqq \alpha .
$$

Because $f^{\prime}$ possesses the Darboux property, we infer

$$
\sup _{x \in I \cdot E} f^{\prime}(x)=\beta, \quad \inf _{x \in I \cdot E} f^{\prime}(x)=\alpha,
$$

so that we have now shown that, considered on domain $E$, and so a fortiori on domain $\bar{E}$, the function $f^{\prime}$ is discontinuous at each point of $E$. On domain $\bar{E}$ the saltus of $f^{\prime}$ at each point of $E$ has been shown to be at least $\beta-\alpha$; the same, then, will be true at each point of $\bar{E}$. The function $f^{\prime}$ has now been shown to be everywhere discontinuous considered on domain $\bar{E}$; this completes the proof.

The University of Pennsyluania

${ }^{3}$ Kuratowsky, Topologie, Warsaw, 1933, p. 189. 